\title{
EuroPVM/MPI Full-Day Tutorial. Using MPI-2: A Problem-Based Approach ${ }^{\star}$
}

\author{
William Gropp ${ }^{1}$ and Ewing Lusk ${ }^{2}$ \\ ${ }^{1}$ University of Illinois \\ wgropp@illinois.edu \\ 2 Argonne National Laboratory \\ lusk@mcs .anl.gov
}

\begin{abstract}
MPI-2 introduced many new capabilities, including dynamic process management, one-sided communication, and parallel I/O. Implementations of these features are becoming widespread. This tutorial shows how to use these features by showing all of the steps involved in designing, coding, and tuning solutions to specific problems. The problems are chosen for their practical use in applications as well as for their ability to illustrate specific MPI-2 topics. Complete examples that illustrate the use of MPI one-sided communication, MPI parallel I/O, and hybrid programming with MPI and threads will be discussed and full source code will be made available to the attendees. Each example will include a hands-on lab session; these sessions will also introduce the use of performance and correctness debugging tools that are available for the MPI environment. Guidance on tuning MPI programs will be included, with examples and data from MPI implementations on a variety of parallel systems, including Sun, IBM, SGI, and clusters. Examples in C, Fortran, and $\mathrm{C}++$ will be included. Familiarity with basic MPI usage will be assumed.
\end{abstract}

\footnotetext{
* This work was supported by the U.S. Dept. of Energy under Contract DE-AC02-
} $06 \mathrm{CH} 11357$. 